\title{
Quantum Phase Transition between a Topological and a Trivial Semimetal from Holography
}

\author{
Karl Landsteiner, ${ }^{*}$ Yan Liu, ${ }^{\dagger}$ and Ya-Wen Sun \\ Instituto de Física Teórica UAM/CSIC, C/Nicolás Cabrera 13-15, \\ Universidad Autónoma de Madrid, Cantoblanco, 28049 Madrid, Spain \\ (Received 2 December 2015; revised manuscript received 1 February 2016; published 25 February 2016)

\begin{abstract}
We present a holographic model of a topological Weyl semimetal. A key ingredient is a time-reversal breaking parameter and a mass deformation. Upon varying the ratio of mass to time-reversal breaking parameter the model undergoes a quantum phase transition from a topologically nontrivial semimetal to a trivial one. The topological nontrivial semimetal is characterized by the presence of an anomalous Hall effect. The results can be interpreted in terms of the holographic renormalization group (RG) flow leading to restoration of time reversal at the end point of the RG flow in the trivial phase.
\end{abstract}

DOI: 10.1103/PhysRevLett.116.081602

Weyl semimetals are an exciting new class of 3D materials with exotic transport properties [1,2]. They are characterized by pointlike singularities in the Brillouin zone at which conduction and valence bands touch. Around these points the electronic quasiparticle excitations can be described by either left- or right-handed Weyl spinors. The Nielsen-Ninomiya theorem guarantees that left- and righthanded Weyl spinors always appear in pairs [3]. When time-reversal symmetry is broken the left- and right-handed quasiparticles can sit at different points in the Brillouin zone. Effectively, the Weyl fermions are separated by an (axial) vector in the momentum space. The wave function of a Weyl spinor can be understood as a monopole of the Berry curvature in momentum space. Left-handed Weyl fermions have monopole charge +1 and the right-handed ones have monopole charge -1 [4-6]. Since the monopole charge in momentum space is a topological invariant it is still present in fermionic two-point correlation functions when interactions are taken into account [7]. However, at strong coupling such semiclassical reasoning based on fermionic wave functions or correlators might not be available and the dynamical variables are physical operators with the quantum numbers of fermion bilinears. The question arises then if it is possible to construct a model at strong coupling that has the essential physical properties of a Weyl semimetal, in particular, if there exists any strongly coupled model in which a quantum phase transition between a topological and a topologically trivial state persists even in the absence of the notion of singularities in the dispersion relations of fermionic two-point correlations functions? A tool to answer these questions is the AdS/CFT correspondence ("holography"). It has already proved to be extremely useful for the understanding of strongly correlated relativistic systems, including superconductors [8], strange metals [9,10], lattice systems [11], etc. In particular, the modern understanding of anomaly related transport phenomena, such as the chiral magnetic and chiral vortical effects, is based to a considerable part on research using holographic models [12-15].

We first review a quantum field theoretical model with the same local properties around the band touching points as a Weyl semimetal,

$$
\mathcal{L}=\bar{\Psi}\left(i \not \partial-e A-\gamma_{5} \vec{\gamma} \cdot \vec{b}+M\right) \Psi
$$

It takes the form of a "Lorentz breaking" Dirac system [18] with Lagrangian (1). Here $X=\gamma^{\mu} X_{\mu}, A_{\mu}$ is the electromagnetic gauge potential, $\gamma^{\mu}$ are the Dirac matrices, and $\gamma_{5}=i \gamma_{0} \gamma_{1} \gamma_{2} \gamma_{3}$ allows us to define left- or right-handed spinors via $\left(1 \pm \gamma_{5}\right) \Psi=\Psi_{L, R}$. Without loss of generality we take $\vec{b}=b \hat{e}_{z}$.

The spectrum of Eq. (1) is sketched in Fig. 1. As long as $|b|>|M|$ the spectrum is ungapped. It is characterized by band inversion and at the crossing points the wave function is well described by Weyl fermion. The separation of the Weyl cones is given by $\sqrt{b^{2}-M^{2}}$. In this situation the quantum field theoretical model at low energies can be further reduced to an effective low energy Lagrangian of the form (1) with $M_{\text {eff }}=0$ and $\vec{b}_{\text {eff }}=\sqrt{b^{2}-M^{2}} \hat{e}_{z}$. For $|b|<|M|$ the system is gapped and the low energy description is simply one of a massive Dirac fermions with $b_{\text {eff }}=0$ and $M_{\text {eff }}=\sqrt{M^{2}-b^{2}}$.

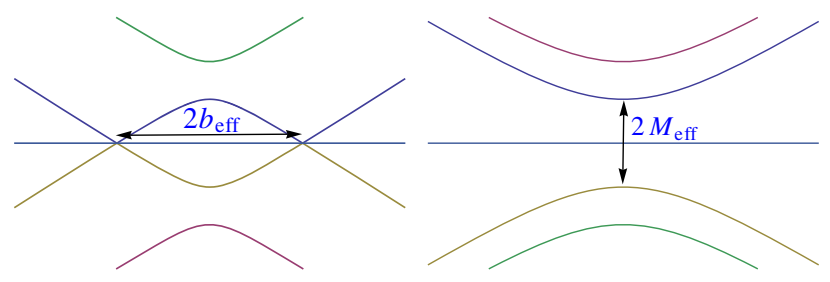

FIG. 1. Left panel: For $b^{2}>M^{2}$ there are two Weyl nodes in the spectrum. They are separated by the distance $\sqrt{b^{2}-M^{2}}$ in momentum space. Right panel: For $b^{2}<M^{2}$ the system is gapped with gap $M_{\text {eff }}=\sqrt{M^{2}-b^{2}}$. 
The axial anomaly

$$
\partial_{\mu} J_{5}^{\mu}=\frac{1}{16 \pi^{2}} \varepsilon^{\mu \nu \rho \lambda} F_{\mu \nu} F_{\rho \lambda}+2 M \bar{\Psi} \gamma_{5} \Psi
$$

implies the anomalous Hall effect [6,19-23]

$$
\vec{J}=\frac{1}{2 \pi^{2}} \vec{b}_{\mathrm{eff}} \times \vec{E} .
$$

Accordingly, the system undergoes a quantum phase transition from the topologically nontrivial Weyl semimetal phase to a trivial insulating phase. This is an example of a topological phase transition not subject to the Landau classification. In both phases the same symmetries are broken by the nonzero values of the couplings $M, b$. Since the topological phase is characterized by the presence of the Hall effect we can take the Hall conductivity as the order parameter of the transition. More generally, additional Dirac fermions might be present. Then the topologically trivial phase would not be gapped but is itself a semimetal. The quantum phase transition is then between a topological and a trivial semimetal. This will be the case in our holographic model.

The anomalous Hall effect arises as a one-loop contribution to the polarization tensor. Regularization ambiguities [24] present in the quantum field theoretical model are resolved either by matching to a tight-binding model $[25,26]$ or by considering anomaly cancellation arising from chiral edge states at boundaries (Fermi arcs) [27]. Essentially, this demands a regularization that is gauge invariant in the presence of axial gauge fields (a source for the axial current).

In the following, we consider the holographic action

$$
\begin{aligned}
S= & \int d^{5} x \sqrt{-g}\left[\frac{1}{2 \kappa^{2}}\left(R+\frac{12}{L^{2}}\right)-\frac{1}{4} F^{2}-\frac{1}{4} F_{5}^{2}\right. \\
& +\frac{\alpha}{3} \varepsilon^{\mu \nu \rho \sigma \tau} A_{\mu}\left(F_{\nu \rho}^{5} F_{\sigma \tau}^{5}+3 F_{\nu \rho} F_{\sigma \tau}\right) \\
& \left.+\left(D_{\mu} \Phi\right)^{*}\left(D^{\mu} \Phi\right)-V(\Phi)\right],
\end{aligned}
$$

where $\kappa$ is the Newton constant, $L$ is the AdS radius, and $\alpha$ is the Chern-Simons coupling constant. In holography, symmetries of the field theory correspond to gauge fields in AdS space. The electromagnetic $U(1)$ symmetry is represented by the AdS bulk gauge field $V_{\mu}$ with field strength $F=d V$. The axial $U(1)$ symmetry is represented by the gauge field $A_{\mu}$ with field strength $F_{5}=d A$ [28]. It is anomalous and the anomaly is represented in Eq. (4) by the Chern-Simons part of the action with coupling constant $\alpha$. The gauge invariant regularization corresponds to this choice of Chern-Simons term. It is the unique one that makes the electromagnetic symmetry nonanomalous [29]. The mass deformation is introduced via a non-normalizable mode of the scalar field $\Phi$ [30]. This scalar field is charged only under the axial gauge transformation and its covariant derivative is $D_{\mu} \Phi=\left(\partial_{\mu}-i q A_{\mu}\right) \Phi$. The scalar field potential is $m^{2}|\Phi|^{2}+(\lambda / 2)|\Phi|^{4}$. The AdS bulk mass $m^{2} L^{2}=-3$ is chosen such that the dual operator has dimension three and its source has dimension one. This matches exactly the dimension of the dual of a mass deformation. The electromagnetic and axial currents [31] are defined as

$$
\begin{gathered}
J^{\mu}=\lim _{r \rightarrow \infty} \sqrt{-g}\left(F^{\mu r}+4 \alpha \varepsilon^{r \mu \beta \rho \sigma} A_{\beta} F_{\rho \sigma}\right), \\
J_{5}^{\mu}=\lim _{r \rightarrow \infty} \sqrt{-g}\left(F_{5}^{\mu r}+\frac{4 \alpha}{3} \varepsilon^{r \mu \beta \rho \sigma} A_{\beta} F_{\rho \sigma}^{5}\right) .
\end{gathered}
$$

The model has been studied before in the probe limit in Ref. [33].

We are looking for solutions that are asymptotically AdS. In addition, the holographic analogues of the mass term and the time-reversal breaking parameters in Eq. (1) are introduced via the boundary conditions at $r=\infty$,

$$
\lim _{r \rightarrow \infty} r \Phi=M, \quad \lim _{r \rightarrow \infty} A_{z}=b .
$$

Our ansatz for the zero temperature solution is

$$
\begin{aligned}
d s^{2} & =u\left(-d t^{2}+d x^{2}+d y^{2}\right)+\frac{d r^{2}}{u}+h d z^{2}, \\
\Phi & =\phi, \quad A=A_{z} d z .
\end{aligned}
$$

Note that due to the conformal symmetry at zero temperature only $M / b$ is a tunable parameter of the system. In the following, we set $2 \kappa^{2}=L=1$.

Critical solution.-The following Lifshitz-type solution is an exact solution of the system.

$$
\begin{aligned}
d s^{2} & =u_{0} r^{2}\left(-d t^{2}+d x^{2}+d y^{2}\right)+\frac{d r^{2}}{u_{0} r^{2}}+h_{1} r^{2 \beta} d z^{2}, \\
A_{z} & =r^{\beta}, \quad \phi=\phi_{0} .
\end{aligned}
$$

It has the anisotropic Lifshitz-type symmetry $\left(t, x, y, r^{-1}\right) \rightarrow$ $s\left(t, x, y, r^{-1}\right)$ and $z \rightarrow s^{\beta} z$. We need to introduce irrelevant deformations to flow it to the UV to match the boundary conditions (7). We can use the scaling symmetry $z \rightarrow s z$ to set the coefficient in $A_{z}$ to be 1 . There are four constants $\left\{u_{0}, h_{1}, \beta, \phi_{0}\right\}$ that are determined by the value of $\lambda, m$, and $q$. We will focus on the simplest case in which there exists only one critical solution and leave the most general $q, \lambda$ analysis for further research. To flow this geometry to asymptotic AdS in the UV, we need to consider the following irrelevant perturbation around the Lifshitz fix point $u=u_{0} r^{2}\left(1+\delta u r^{\alpha}\right), h=h_{1} r^{\beta}\left(1+\delta h r^{\alpha}\right)$, $A_{z}=r^{\beta}\left(1+\delta a r^{\alpha}\right), \phi=\phi_{0}\left(1+\delta \phi r^{\alpha}\right)$. Because of the scaling symmetry, only the sign of $\delta \phi$ is a free parameter and others are fully determined by $\delta \phi= \pm 1$. Numerics shows that only $\delta \phi=-1$ corresponds to asymptotic AdS space at the UV. From now we fix the parameters $q=1, \lambda=1 / 10$. In this case we have 
$\left(u_{0}, h_{1}, \beta, \phi_{0}, \alpha\right) \simeq(1.468,0.344,0.407,0.947,1.315)$ and $(\delta u, \delta h, \delta a) \simeq(0.369,-2.797,0.137) \delta \phi$. We find the critical value $M / b \simeq 0.744$, which corresponds to the transition point.

Topological nontrivial phase.-The second kind of solution [34] at leading order in the IR is

$u=r^{2}, \quad h=r^{2}, \quad A_{z}=a_{1}+\frac{\pi a_{1}^{2} \phi_{1}^{2}}{16 r} e^{-\frac{2 a_{1} q}{r}}$,

$\phi=\sqrt{\pi} \phi_{1}\left(\frac{a_{1} q}{2 r}\right)^{3 / 2} e^{-\frac{a_{1} q}{r}} ;$

$\lambda$ appears only at higher order terms which become important when $M / b$ is close to the critical value. We set $a_{1}$ to a numerically convenient value and we rescale to $b=1$ later on.

Starting from the near horizon solution, we can numerically integrate equations towards the UV and take $\phi_{1}$ as the shooting parameter to get an $\mathrm{AdS}_{5}$ to $\mathrm{AdS}_{5}$ domain wall. For our chosen values of $\lambda$ and $q$ this kind of solution exists only for $M / b<0.744$.

Topological trivial phase.-The third kind of near horizon solution to leading order is

$u=\left(1+\frac{3}{8 \lambda}\right) r^{2}, \quad h=r^{2}, \quad A_{z}=a_{1} r^{\beta_{1}}, \quad \phi=\sqrt{\frac{3}{\lambda}}+\phi_{1} r^{\beta_{2}}$,

where $\quad\left(\beta_{1}, \beta_{2}\right)=\left(\sqrt{1+\left[48 q^{2} /(3+8 \lambda)\right]}-1\right.$, $2 \sqrt{[(3+20 \lambda) /(3+8 \lambda)]}-2)$. For our choice of $\lambda$ and $q$ $\left(\beta_{1}, \beta_{2}\right)=(\sqrt{(259 / 19)}-1,(10 / \sqrt{19})-2)$. We can set $a_{1}$ to be 1 and take $\phi_{1}$ as the shooting parameter to get the $\mathrm{AdS}_{5}$ to $\mathrm{AdS}_{5}$ domain wall. We find that this type of solution only exists for $M / b>0.744$.

In Fig. 2 we show the behavior of the scalar field and the gauge field for all three phases at several different values of $M / b$. For a given value of $M / b$ only one of the three types of solutions exists. Note that the value of the gauge field on the horizon matches continuously between the two phases whereas the value of the scalar field on the horizon jumps discontinuously. Close to the transition point, the near horizon geometry (10) or (11) quickly flows to the critical solution in the intermediate IR region as indicated by the brown and orange curves in Fig. 2. Adding standard holographic counterterms we can also compute the free
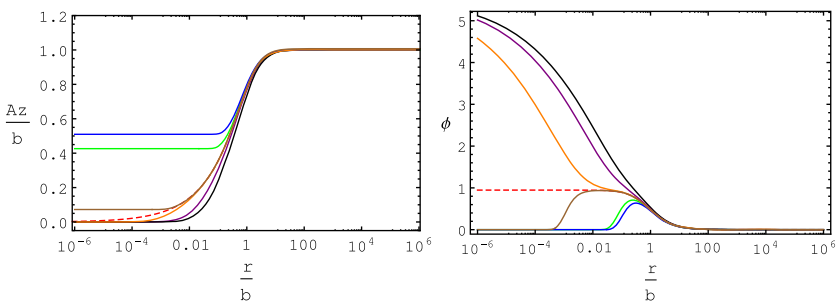

FIG. 2. The bulk profile of background $A_{z}$ and $\phi$ for $M / b=$ 0.695 (blue), 0.719 (green), 0.745 (orange), 0.744 (red-dashed), 0.743 (brown), 0.778 (purple), 0.856 (black). energy density (see the Supplemental Material [37]). We find a continuous and smooth behavior at the critical value. Note that the free energy is independent of the ChernSimons coupling and, consequently, does not probe the topological nature of the transition in contrast to the Hall conductivity.

Finite temperature solutions. - In order to find finite temperature solutions with a regular horizon at a finite value of $r$ we use the ansatz

$$
\begin{aligned}
d s^{2} & =-u d t^{2}+\frac{d r^{2}}{u}+f\left(d x^{2}+d y^{2}\right)+h d z^{2}, \\
\Phi & =\phi, \quad A=A_{z} d z,
\end{aligned}
$$

and the conditions that at $r=r_{0}$ the functions are analytic and that $u$ has simple zero. Using the scaling symmetries of AdS and the constraint from the equations of motion at the horizon $r=r_{0}$ we are left with only two dimensionless parameters. In the UV these are mapped to $M / b$ and $T / b$.

Conductivities.-Conductivities can now be computed with the help of a Kubo formula via retarded correlation functions

$$
\sigma_{m n}=\lim _{\omega \rightarrow 0} \frac{1}{i \omega}\left\langle J_{m} J_{n}\right\rangle(\omega, \vec{k}=0) .
$$

In holography, the retarded Greens functions can be obtained by studying the fluctuations of the gauge fields around the background with infalling boundary conditions at the horizon.

The anomalous Hall conductivity is the off-diagonal part of Eq. (13). We consider the following fluctuations $\delta V_{x}=$ $v_{x}(r) e^{-i \omega t}, \delta V_{y}=v_{y}(r) e^{-i \omega t}$, and define $v_{ \pm}=v_{x}+i v_{y}$,

$$
v_{ \pm}^{\prime \prime}+\left(\frac{h^{\prime}}{2 h}+\frac{u^{\prime}}{u}\right) v_{ \pm}^{\prime}+\frac{\omega^{2}}{u^{2}} v_{ \pm} \pm \frac{8 \omega \alpha}{u \sqrt{h}} A_{z}^{\prime} v_{ \pm}=0
$$

Note that these are the same for the zero and finite temperature backgrounds. To solve these equations we follow the usual near-far matching method to first impose ingoing boundary conditions at the near region solutions and match with the far region solutions at a matching region to give the boundary condition for the far region solutions [41]. To compute the Greens function we normalize the fluctuation to unity at the boundary. The response in the current is then given by $G_{ \pm}=-\left.f \sqrt{h} u v_{ \pm}^{\prime}\right|_{r=\infty} \pm 8 \alpha b \omega$. Note that the second term stems from the Chern-Simons current in Eq. (5). We only need to compute the leading order in $\omega$. For both cases $T=0$ and $T>0$ we can express the result as

$\sigma_{x y}=\frac{G_{+}-G_{-}}{2 \omega}=8 \alpha A_{z}\left(r_{0}\right), \quad \sigma_{x x}=\sigma_{y y}=\sqrt{h\left(r_{0}\right)}$.

We emphasize that for $T=r_{0}=0$ and $h(0)=0$ the diagonal conductivities vanish at zero temperature. The anomalous Hall effect (see Fig. 3) is determined by 


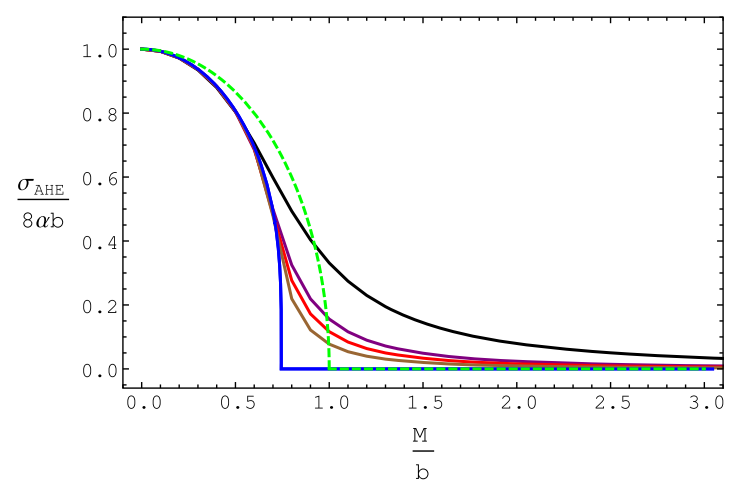

FIG. 3. Anomalous Hall conductivity for different temperatures. The solid lines correspond to our holographic model. For $T=0$ there is a sharp but continuous phase transition at a critical value of $M / b$ (blue), which becomes a smooth crossover at $T>0$. We show the curves for $T / b=0.1$ (black), $0.05 b$ (purple), 0.03 (brown). For comparison, we also show the result for the weak coupling model as a dashed (green) line. Near the transition the Hall conductivity behaves as $\sigma_{x y} \propto\left[(b / M)_{c}-b / M\right]^{\alpha}$ with $\alpha \approx 0.211$ (to be contrasted with the field theory model for which $\alpha=0.5$ ).

the IR value of the axial gauge field. We can identify $b_{\text {eff }}=A_{z}(r=0)$. At zero temperature it is nonvanishing only in the second type of solutions described above. We thus call this the topological nontrivial solution. The third kind of zero temperature solution is characterized by the restoration of time-reversal invariance at the end point of the holographic renormalization group (RG) flow $A_{z}(0)=0$.

Longitudinal conductivity.-The longitudinal electric conductivity at both finite and zero temperature can be computed from the fluctuation $\delta V_{z}=v_{z} e^{-i \omega t}$ with equation of motion

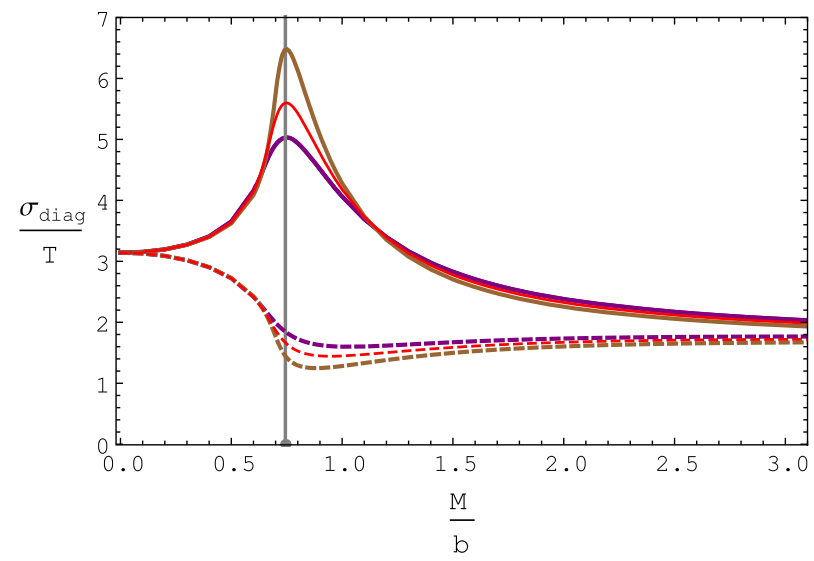

FIG. 4. The transverse and longitudinal electric conductivities for different temperatures. The solid lines are for $\sigma_{x x}=\sigma_{y y}$ and the dashed lines are for $\sigma_{z z}$ from our holographic model with $T / b=0.05$ (purple), 0.04 (red), 0.03 (brown). The gray line is the critical value of $M / b$ at the topological phase transition.

$$
v_{z}^{\prime \prime}+\left(\frac{f^{\prime}}{f}-\frac{h^{\prime}}{2 h}+\frac{u^{\prime}}{u}\right) v_{z}^{\prime}+\frac{\omega^{2}}{u^{2}} v_{z}=0 .
$$

At zero temperature we substitute $f=u$. We again solve it using the semianalytic method of near-far region matching. At zero temperature we again find $\sigma_{z z}=0$ and for finite temperature we find

$$
\sigma_{z z}=\left.\frac{f}{\sqrt{h}}\right|_{r=r_{0}} .
$$

The three types of background solutions can now be classified according to the anomalous Hall effect (AHE). There is a phase for $M / b$ smaller than a critical value in which the axial gauge field flows along the holographic direction towards a constant but nonzero value in the IR. The end point of this holographic flow of the axial gauge field determines the Hall conductivity $\sigma_{x y}$ (or $\sigma_{\mathrm{AHE}}$ ). At $M=0$ the flow is trivial and the Hall response is completely determined by the Chern-Simons current at the boundary of AdS space. For $M \neq 0$ a nontrivial flow develops, the Hall conductivity has now two parts, a dynamical part, that can only be determined by solving the Eqs. (14) and the Chern-Simons part determined by the boundary values of the fields. At the critical value (for our choice of parameters this is $\left.(M / b)_{\mathrm{c}} \simeq 0.744\right)$ the Hall conductivity vanishes. At this value there is a critical solution with a nontrivial scaling exponent in the $z$ direction. For even larger values of $M / b$ the solution shows no Hall effect. The axial gauge field flows to $A_{z}=0$ in the far IR. In contrast, now the scalar field obtains a nontrivial IR value. This corresponds to the effect that the cosmological constant has a different value in the far IR; i.e., the trivial solution is a domain wall in $\mathrm{AdS}$ similar to the zero temperature superconductor solutions described in Ref. [35]. Since in holography the cosmological constant is a measure of the effective number of degrees of freedom, we interpret the trivial solution as one in which some of the UV degrees of freedom are gapped out along the RG flow. We have thus found a holographic zero temperature quantum phase transition between a topological phase characterized by a nonvanishing Hall conductivity and a topological trivial phase with zero Hall conductivity. All diagonal conductivities vanish at zero temperature.

At $T \neq 0$ the quantum phase transition becomes a smooth crossover behavior. The far IR physics is covered by a horizon at some finite value of the holographic coordinate. It is also interesting to observe the behavior of the diagonal conductivities at finite $T$ as a function of $M / b$ (see Fig. 4). We see that the transverse diagonal conductivities develop a peak roughly at the critical value whereas the longitudinal one develops a minimum. The height of the peak and the depth of the minimum grow with temperature. At $M=0$ we simply have $\sigma_{x x, y y, z z}=\pi T$ and for large $M$ the conductivities tend to a value 
$\sigma_{x x, y y, z z}=c \pi T$ with $c<1$ and independent of temperature. This is consistent with the interpretation that some but not all degrees of freedom are gapped out in the trivial phase and that the phase transition is between a topological semimetal and a trivial semimetal.

We believe our model can serve as a starting point to investigate the paradigm on topological ungapped states of matter in holography and, more generally, without resorting to the concept of Berry curvature of fermionic wave functions in momentum space.

We thank R. G. Cai, A. Cortijo, Y. Ferreiro, S. Hartnoll, D. Kharzeev, V. Jacobs, M. A. H. Vozmediano, and J. Zaanen for useful discussions. This work has been supported by Project No. FPA2012-32828 and by the Centro de Excelencia Severo Ochoa Programme under Grant No. SEV-2012-0249. The work of Y. W. S. was also supported by the European Union through a Marie Curie Individual Fellowship MSCA-IF-2014-659135. Y. L. and Y. W. S. would like to thank KITPC for its hospitality and its partial support during the program "Holographic duality for condensed matter physics."

*karl.landsteiner@csic.es

†yanliu.th@gmail.com

*yawen.sun@csic.es

[1] O. Vafek and A. Vishwanat, Dirac fermions in solids: From high-Tc cuprates and graphene to topological insulators and Weyl semimetals, Annu. Rev. Condens. Matter Phys. 5, 83 (2014).

[2] P. Hosur and X. Qi, Recent developments in transport phenomena in Weyl semimetals, C.R. Phys. 14, 857 (2013).

[3] H. B. Nielsen and M. Ninomiya, The Adler-Bell-Jackiw anomaly and Weyl fermions in a crystal, Phys. Lett. B 130, 389 (1983).

[4] E. Kiritsis, A topological investigation of the quantum adiabatic phase, Commun. Math. Phys. 111, 417 (1987).

[5] G. E. Volovik, The Universe in a Helium Droplet (Oxford University Press, Oxford, 2003).

[6] F. D. M. Haldane, Berry Curvature on the Fermi Surface: Anomalous Hall Effect as a Topological Fermi-Liquid Property, Phys. Rev. Lett. 93, 206602 (2004).

[7] W. Witczak-Krempa, M. Knap, and D. Abanin, Interacting Weyl Semimetals: Characterization via the Topological Hamiltonian and its Breakdown, Phys. Rev. Lett. 113, 136402 (2014).

[8] S. A. Hartnoll, C. P. Herzog, and G. T. Horowitz, Building a Holographic Superconductor, Phys. Rev. Lett. 101, 031601 (2008).

[9] H. Liu, J. McGreevy, and D. Vegh, Non-Fermi liquids from holography, Phys. Rev. D 83, 065029 (2011).

[10] M. Cubrovic, J. Zaanen, and K. Schalm, String theory, Quantum phase transitions and the emergent Fermi-liquid, Science 325, 439 (2009).

[11] G. T. Horowitz, J. E. Santos, and D. Tong, Optical Conductivity with holographic lattices, J. High Energy Phys. 07 (2012) 168.
[12] J. Erdmenger, M. Haack, M. Kaminski, and A. Yarom, Fluid dynamics of R-charged black holes, J. High Energy Phys. 01 (2009) 055.

[13] N. Banerjee, J. Bhattacharya, S. Bhattacharyya, S. Dutta, R. Loganayagam, and P. Surowka, Hydrodynamics from charged black branes, J. High Energy Phys. 01 (2011) 094.

[14] K. Landsteiner, E. Megias, L. Melgar, and F. Pena-Benitez, holographic gravitational anomaly and chiral vortical effect, J. High Energy Phys. 09 (2011) 121.

[15] Previous holographic approaches to the physics of Weyl semimetals $[16,17]$ differ from our approach in that they study holographic fermionic spectral functions.

[16] V. P. J. Jacobs, S. J. G. Vandoren, and H. T. C. Stoof, Holographic interaction effects on transport in Dirac semimetals, Phys. Rev. B 90, 045108 (2014).

[17] U. Gursoy, V. Jacobs, E. Plauschinn, H. Stoof, and S. Vandoren, Holographic models for undoped Weyl semimetals, J. High Energy Phys. 04 (2013) 127.

[18] D. Colladay and V. A. Kostelecky, Lorentz violating extension of the standard model, Phys. Rev. D 58, 116002 (1998).

[19] K. Y. Yang, Y. M. Lu, and Y. Ran, Quantum Hall effects in a weyl semimetal: Possible application in pyrochlore iridates, Phys. Rev. B 84, 075129 (2011).

[20] G. Xu, H. Weng, Z. Wang, X. Dai, and Z. Fang, Chern Semi-Metal and Quantized Anomalous Hall Effect in $\mathrm{HgCr}_{2} \mathrm{Se}_{4}$, Phys. Rev. Lett. 107, 186806 (2011).

[21] A. A. Burkov and L. Balents, Weyl Semimetal in a Topological Insulator Multilayer, Phys. Rev. Lett. 107, 127205 (2011).

[22] A. A. Zyuzin and A. A. Burkov, Topological response in Weyl semimetals and the chiral anomaly, Phys. Rev. B 86, 115133 (2012).

[23] Y. Chen, S. Wu, and A. A. Burkov, Axion response in Weyl semimetals, Phys. Rev. B 88, 125105 (2013).

[24] R. Jackiw, When radiative corrections are finite but undetermined, Int. J. Mod. Phys. B 14, 2011 (2000).

[25] A. G. Grushin, Consequences of a condensed matter realization of Lorentz violating QED in Weyl semi-metals, Phys. Rev. D 86, 045001 (2012).

[26] M. M. Vazifeh and M. Franz, Electromagnetic Response of Weyl Semimetals, Phys. Rev. Lett. 111, 027201 (2013).

[27] P. Goswami and S. Tewari, Axionic field theory of $(3+1)-$ dimensional Weyl semimetals, Phys. Rev. B 88, 245107 (2013).

[28] Note the different conventions from here on.

[29] A. Rebhan, A. Schmitt, and S. A. Stricker, Anomalies and the chiral magnetic effect in the Sakai-Sugimoto model, J. High Energy Phys. 01 (2010) 026; A. Gynther, K. Landsteiner, F. Pena-Benitez, and A. Rebhan, Holographic anomalous conductivities and the chiral magnetic effect, J. High Energy Phys. 02 (2011) 110.

[30] A. Jimenez-Alba, K. Landsteiner, Y. Liu, and Y. W. Sun, Anomalous magnetoconductivity and relaxation times in holography, J. High Energy Phys. 07 (2015) 117.

[31] These are the consistent currents. Imposing the equations of motion the vector current $J^{\mu}$ is conserved, whereas the conservation of the axial current $J_{5}^{\mu}$ is broken by the scalar field and by the anomaly [30]. It is also possible to define covariant currents by dropping the Chern-Simons terms [32]. 
[32] W. A. Bardeen and B. Zumino, Consistent and covariant anomalies in gauge and gravitational theories, Nucl. Phys. B244, 421 (1984).

[33] K. Landsteiner and Y. Liu, The holographic Weyl semimetal, Phys. Lett. B 753, 453 (2016).

[34] Similar near-horizon geometries were found in Refs. [35,36] in the context of holographic superconductors.

[35] S. S. Gubser and A. Nellore, Ground states of holographic superconductors, Phys. Rev. D 80, 105007 (2009).

[36] P. Basu, J. He, A. Mukherjee, and H. H. Shieh, Hard-gapped holographic superconductors, Phys. Lett. B 689, 45 (2010).

[37] See Supplemental Material at http://link.aps.org/ supplemental/10.1103/PhysRevLett.116.081602 for text, equations, and the figure supporting statements made in the Letter, which includes Refs. [38-40].

[38] V. Balasubramanian and P. Kraus, A Stress tensor for Antide Sitter gravity, Commun. Math. Phys. 208, 413 (1999).

[39] R. C. Myers, Stress tensors and Casimir energies in the AdS/ CFT correspondence, Phys. Rev. D 60, 046002 (1999).

[40] S. de Haro, S. N. Solodukhin, and K. Skenderis, Holographic reconstruction of space-time and renormalization in the AdS/CFT correspondence, Commun. Math. Phys. 217, 595 (2001).

[41] T. Faulkner, H. Liu, J. McGreevy, and D. Vegh, Emergent quantum criticality, Fermi surfaces, and $\operatorname{AdS}(2)$, Phys. Rev. D 83, 125002 (2011). 